\title{
Viscoelastic behavior of allografts and scaffolds composed of extracellular matrices
}

\begin{abstract}
Collagen is the major structural fiber found in mammalian tissues. It is a protein in the form of a triple-helix that is found in several subfamilies, the most abundant of which is the fiber-forming group containing Types I, II and III. Type I collagen is found in tendons, skin, cornea, bone, lung and vessel walls. This collagen is thought to give rise to the high tensile strengths of collagen fibers in tissues; in addition, it is actively involved in other physiologic processes such mechanotransduction. However, the nonlinear mechanical behavior and viscoelasticity of collagen fibers make analysis of the mechanical properties of tissues complicated.
\end{abstract}

Mechanistically, during mechanical loading, a tensional increase in the D period is observed with increasing strain that is associated with:

A. Molecular elongation at the triple-helical level of structure;

B. Increases in the gap distance between the end of one triple-helix and the start of the next one in the microfibril; and

C. Molecular slippage.

The state of mechanical loading of tissues dictates the regulation of tissue metabolism by up- or down-regulating mechanotransduction through several mechanisms including the phosphorelay pathways.

In this paper, we discuss the relationship between collagen hierarchical structure and its viscoelastic mechanical properties. Using vibrational analysis and optical coherence tomography it is hoped that the mechanical properties of collagenous tissues can be studied in vivo in order to better understand tissue mechanics and to be better able to offer early diagnosis and differentiation of different disease states.

Keywords: collagen fibers, mechanical properties, optical coherence tomography, vibrational analysis, tendon, skin, cartilage, elastic arteries
Volume 2 Issue I - 2017

\author{
Frederick H Silver,' Lisa L Silver,' Ruchit G \\ Shah $^{3}$ \\ 'Department of Pathology and Laboratory Medicine, Rutgers, \\ Robert Wood Johnson Medical School, USA \\ ${ }^{2}$ OptoVibronex, LLC, Mt. Bethel, PA 18343, USA \\ ${ }^{3}$ Graduate Program in Biomedical Engineering, Rutgers, The \\ State University of New Jersey, USA
}

\author{
Correspondence: Frederick H Silver, Department of \\ Pathology and Laboratory Medicine, Robert Wood Johnson \\ Medical School, Rutgers, the State University of New Jersey, 675 \\ Hoes Lane, Piscataway, NJ 08854, USA, \\ Email fhsilver@hotmail.com
}

Received: February 01, 2017 | Published: February 20, 2017
Abbreviations: ECM, extracellular matrix; OCT, optical coherence tomography

\section{Introduction}

Collagen fibers form the basic structural components of the extracellular matrix (ECM) of vertebrates that serve to: store elastic energy during muscular deformation, transmit stored energy into joint movement, and transfer excess energy from the joint back to the attached muscles for dissipation. ${ }^{1}$ They also act as mechanotransducers by transferring stress borne by the musculoskeleton to the attached cells in order to regulate tissue metabolism, either up- or down, as a result of changes in mechanical loading. ${ }^{2}$ The viscoelastic behavior of ECM is very important in understanding the tissue reaction to implanted allografts and tissue engineered materials. It is known that excessive stress at the implant-tissue interface leads to intimal hyperplasia with vascular grafts as well as fat tissue necrosis associated with the pressure produced with breast implants and fat necrosis at the femoral catheter site after use of pressure to achieve closure. ${ }^{3}$

For these reasons collagen fiber mechanical behavior in ECMs is intimately related to tissue reactions at the implant-tissue interface. However, analysis of the basic mechanisms behind this behavior is complicated by the viscoelasticity of collagenous tissues, the presence of other components besides collagen, and mechanotransduction processes. The viscoelasticity of collagenous tissues leads to nonlinear behavior; however, recent studies have identified methods to analyze this behavior. ${ }^{2,-6}$ In this paper, we will review the relationship between ECM molecular and hierarchical structures, mechanical properties and mechanotransduction to better understand events that occur at the implant-host tissue interface.

\section{Collagen molecular structure}

Collagen is a protein in the form of a triple-helix that is found in several subfamilies the most abundant of which is the fiber-forming group containing Types I, II and III. Types I, II, and III form the basic structural units of collagen fibrils and fibers along with some minor types of collagen including types V, IX and XII. ${ }^{7}$ Type I collagen is found in tendons, skin, cornea, bone, lung and vessel walls. ${ }^{8}$ This collagen is thought to give rise to the high tensile strengths of collagen fibers in tissues; in addition, it actively is involved in other physiologic processes such mechanotransduction through integrin dependent activation of the phosphorelay pathways. ${ }^{5}$ The activation of the phosphorelay pathways by changes in mechanical stresses on collagen fibers lead to up- or down regulation of protein synthesis and cell division that modulates the events that occur at the implant-tissue interface. Viscoelasticity and mechanotransduction have been studied extensively in tissues such as skin and tendon and therefore we will explore these properties further. 
Tendons and skin have been studied in vitro and exhibit non-linear viscoelastic stress-strain behaviors (Figure 1). ${ }^{9-12}$ The non-linear behavior is derived from the structural hierarchy of these tissues and the domain structure of the collagen triple helix (Figure 2). Viscoelasticity is related to the molecular and fibrillar deformation patterns of collagen fibrils and fibers as discussed below.

Collagen, when stained with heavy metals, in tissues is recognized by transmission microscopy by its regular repeat pattern of the charged amino acid residues. In the quarter-staggered packing pattern, the amino acid sequence of five molecules in cross-section is repeated every $64 \mathrm{~nm}$, a distance termed the D period (Figure 2). The D period varies from about 64 to $67 \mathrm{~nm}$ depending on the tissue of origin. In tendon, the D period is about $64 \mathrm{~nm}$ and in skin it is about $67 \mathrm{~nm}$. Collagen microfibrils in tissues are packed into a quasi-hexagonal array that forms fibrils and fascicles in tendon.

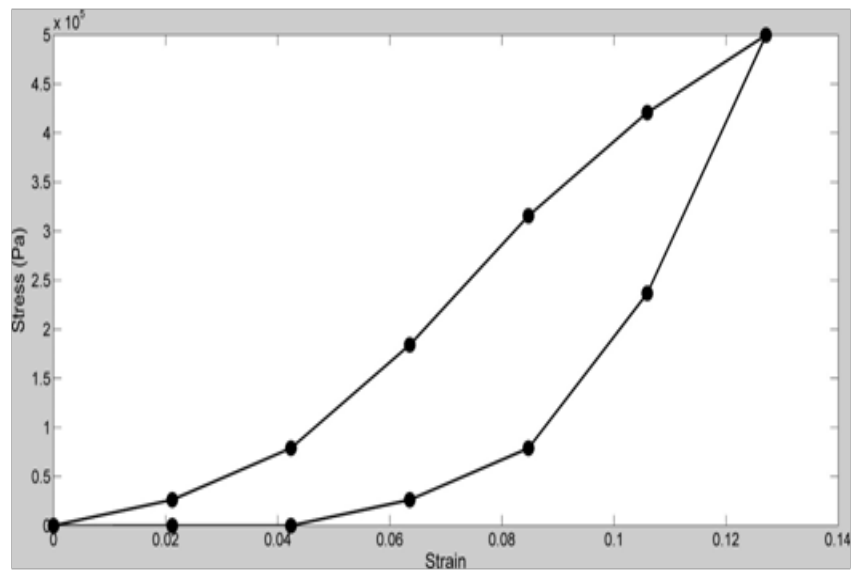

Figure I Typical stress-strain curve for decellularized human dermis tested in uniaxial tension. The dermis is loaded in tension in strain increments and the force at each strain is recorded before another strain increment is added. The loading curve is above the unloading curve and the sample returns to zero stress and zero strain after a recovery period of about 30 minutes. Nonlinearity is a result of several factors including fiber orientation, loading of other components at low strains (elastic fibers) and viscoelasticity. The time dependence of recovery after unloading is a manifestation of viscoelasticity.

\section{Collagen crosslinking}

In fibril forming collagens, the ability to store, transmit and dissipate energy requires crosslink formation between molecules within a microfibril and between microfibrils and other structural units. These crosslinks include lysine and hydroxylysine derivatives and other amino acid residues including histidine that are at the ends of the molecules (Figure 2). ${ }^{4}$ Additional crosslinking also occurs during aging and involves glucose molecules. ${ }^{7}$ The stiffening and poor energy dissipation of collagen fibers associated with aging of the skin involves collagen fiber fragmentation by exposure to UV light and glucose derived crosslinking. In the absence of crosslinking, the mechanical behavior of collagenous materials is primarily viscous with little energy storage. ${ }^{3}$

\section{Viscoelastic behavior of collagen fibers}

Viscoelasticity of collagenous tissues may be important in resisting impact loads especially in the musculoskeleton, however, it complicates the understanding of ECM behavior since most real-time measurements made on these tissues contain both elastic and viscous contributions. ${ }^{11}$ The elastic behavior varies from as high as about $75 \%$ of the total stress for tendon to as low as about $50 \%$ for skin depending on the collagen fiber orientation, rate of loading, degree of crosslinking and the quantities of other tissue constituents. ${ }^{11}$

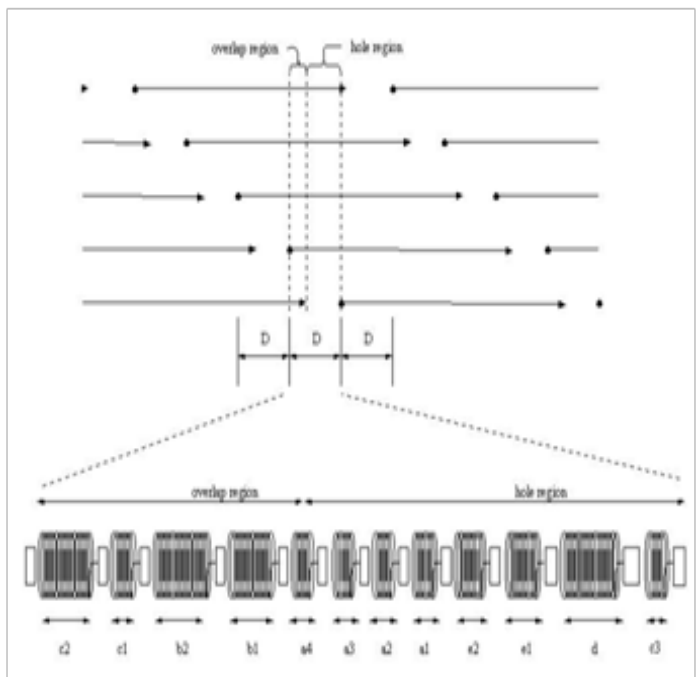

Figure $\mathbf{2}$ The top portion of this figure illustrates the structure of a five membered microfibrillar unit that is believed to be the repeat unit found in collagen fibrils and fibers. In this packing pattern, five collagen molecules are staggered by about $22 \%$ of the molecular length of $300 \mathrm{~nm}$ with respect to their nearest neighbors. A space or hole $0.6 \mathrm{D}$ in length ( $D$ is about $67 \mathrm{~nm}$ ) is left between neighboring molecules. The collagen molecule is $4.4 \mathrm{D}$ long where $\mathrm{D}$ is the stagger between neighboring collagen molecules. The distance $D$ consists of a overlap zone of $0.4 \mathrm{D}$ and a hole region of $0.6 \mathrm{D}$ as is shown by the vertical dotted lines that are superimposed on the microfibril in the diagram. The overlap and hole regions that make up the $D$ repeat consist of 13 rigid and 12 flexible domains and are depicted by the rectangles and springs shown, respectively. The 12 flexible regions are identical to the 12 bands denoted c2 through c3 that are seen as dark vertical lines across the collagen fibril when collagen is stained with heavy metals and viewed in the electron microscope. The twelve flexible regions are believed to be stretched when collagen fibrils are initially mechanically deformed. Collagen molecules in tendon are held together in the microfibril with crosslinks that are at the ends of each molecule from the end on one molecule (see arrows) to the tail of the neighboring molecule (see circles) in the overlap region. This figure was adapted from Silver ${ }^{2}$

A number of excellent studies have been published that have helped in the interpretation of the viscoelastic behavior of tendon and other tissues at the molecular and fibrillar levels. Much of our current understanding of the relationship between hierarchical structure and viscoelastic behavior of ECMs is based on studies of the mechanical properties of developing and mature tendons. ${ }^{13-16}$ The collagen fibril length appears to be important for energy storage and for viscous energy loss due to molecular and fibrillar deformation during tensile deformation.

Mechanistically, during mechanical loading, a tensional increase in the D period is observed with increasing strain that is associated with:

A. molecular elongation at the triple-helical level of structure in the flexible spring-like regions (Figure 2); and

B. Increases in the gap distance between the end of one triple-helix and the start of the next one in the microfibril; these two processes are reversible and result in energy storage. In addition, tension causes some molecular slippage ${ }^{17}$ that may result in viscous energy loss. Molecular stretching occurs at lower stresses followed by increases in the gap spacing and 
molecular sliding that occur at higher stresses. ${ }^{18}$ Molecular stretching and slippage occurs at low strains and is reversible over time. This would explain the time required for recovery of the unloading part of the stress-strain curve (Figure 1). It is believed that molecular stretching occurs first in the regions devoid of proline and hydroxyproline as diagrammatically represented by the springs in Figure 2. These springs represent the charged regions of the sub-bands in the $\mathrm{D}$ period that are shown in transmission electron micrographs of collagen fibrils in tissues. At higher strains, the stress-strain behavior in tension becomes irreversible and collagenous materials fail by defibrillation. ${ }^{2}$

The time-dependent behavior of collagenous materials makes it difficult to interpret stress-strain relationships for these tissues. However using incremental stress-strain curves, the elastic and viscous behaviors can be separated and analyzed in terms of tissue structure. ${ }^{11}$ The viscoelastic properties of ECMs have been obtained by constructing incremental stress-strain curves for a variety of tissues including tendon ${ }^{2,11}$ (Figure 3). Such incremental stress-strain curves are derived for tendon and other ECMs by stretching the tissue in a series of strain increments and then allowing the stress to relax to an equilibrium value at each strain increment before another strain increment is added (Figure 3 top). ${ }^{11}$ By subtracting the elastic stress (equilibrium stress value) from the initial or total stress value, the viscous stress is obtained. By plotting the equilibrium stress versus strain and the total stress minus the equilibrium stress versus strain (Figure 3bottom) we get elastic and viscous stress-strain curves for tendon. ${ }^{2}$ (Figure 3 ). From these curves and the literature, important information can be obtained concerning the mechanism of stretching and sliding of the collagen molecules and fibrils that make up the structure of tendon fibers. ${ }^{2}$ Note in the absence of the crimp the relationship between elastic stress and strain is approximately linear (Figure 4). It turns out that the slope of the elastic stress-strain curve is proportional to the elastic modulus of the collagen molecule, ${ }^{2,19}$ while the viscous stress at a particular strain is a measure of the fibril length., ${ }^{2,19}$ The problem with conducting true incremental stress-strain testing on collagenous materials is that it takes up to 24 hours for the stress to relax to an equilibrium value, before another strain increment can be added. However, the value of doing such an experiment is that it has been reported that the elastic modulus is strain-rate independent for a variety of collagenous tissues and self-assembled collagen fibers. ${ }^{2}$ However, construction of an incremental stress-strain curve is a very tedious task and other methods need to be developed to obtain the elastic modulus for collagenous materials both in vitro and in vivo.

\section{Non-destructive methods for studying viscoelasticity of collagen tissues and scaffolds}

The ability to monitor the mechanical properties of ECMs and implants in vivo is an important measurement needed for early diagnosis of disease, the ability to follow disease progression and to evaluate healing after implantation of a medical device. ${ }^{12}$ Physicians daily "palpate" changes in the properties of tissues associated with tumors and calcification suggesting that there are major changes in the structure and properties of collagen and ECMs during disease processes. It is essential that clinicians be able to assess the changes at the collagen fibril and fiber levels of structure to accurately diagnose and treat diseases such as cancer as well as to follow the wound healing responses to tissue engineered materials and implants.

The use of vibrational analysis in concert with OCT allows one to measure the resonant frequency and mechanical properties of ECMs and implants non-destructively and non-invasively (Figures 5) (Figure 6) at different strains. ${ }^{6,12}$ Since the measurements at different strains using vibrational analysis yield different resonant frequencies, this data can be used to calculate the elastic modulus from incremental testing of ECMs and implants relatively quickly. It is important to match implant mechanical properties with that of the host tissue to reduce the degree of up-regulation of mechanotransduction. Upregulation of mechanotransduction may lead to increases in capsule formation and tissue hyperplasia at the tissue-implant interface Of primary concern besides matching the elastic behavior of ECMs and implants, is that any excess energy imparted at the interface has to be either transmitted away from the interface or dissipated as heat. While the energy that goes into elastic behavior can be transduced into new protein synthesis and cell division, the viscous portion of the energy can only be dissipated as heat or mechanical movement at the tissueimplant interface.
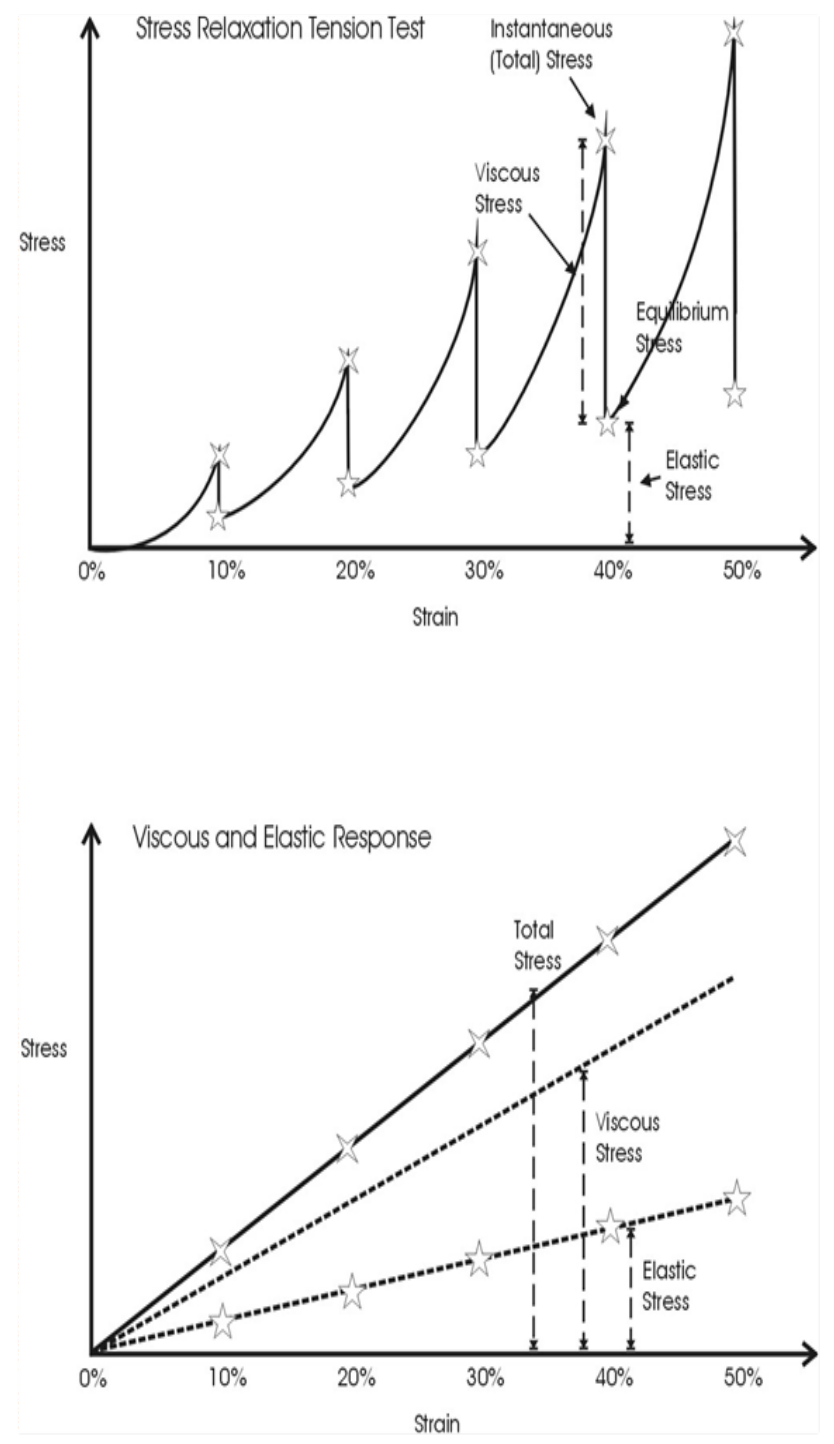

Figure 3 Incremental stress-strain curves for ECMs tested in tension. (Top) A strain increment is applied to the ECM and the initial stress is measured. The strain increment varies from about $2 \%$ for tendon to about $10 \%$ for skin. The stress is allowed to relax at room temperature until an equilibrium value is reached. The process is repeated until the sample fails. (Bottom) Plots of all the initial (total) and equilibrium stresses are made versus strain as well as plot of the total minus equilibrium stress versus strain. The equilibrium stress versus strain curve is equivalent to the elastic stress-strain curve while the difference between the total and equilibrium stress is the viscous stress. 


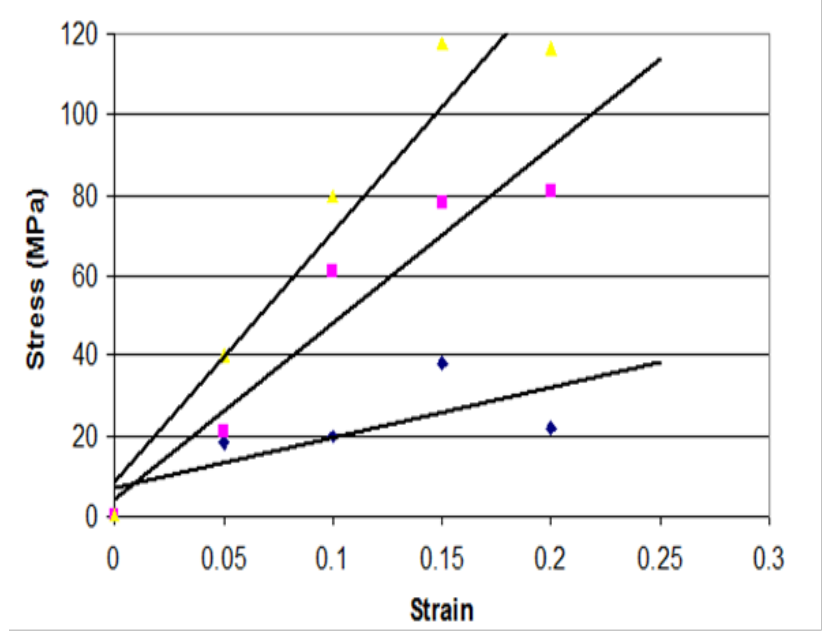

Figure 4 Total, elastic and viscous stress-strain curves for rat tail tendon fibers obtained after incremental stress-strain testing in uniaxial tension. This figure shows that the elastic stress-strain curve (squares) is approximately linear with strain is above the viscous curve (triangles). This diagram illustrates that more energy is stored during tensile deformation of tendon than is dissipated as heat during stretching.

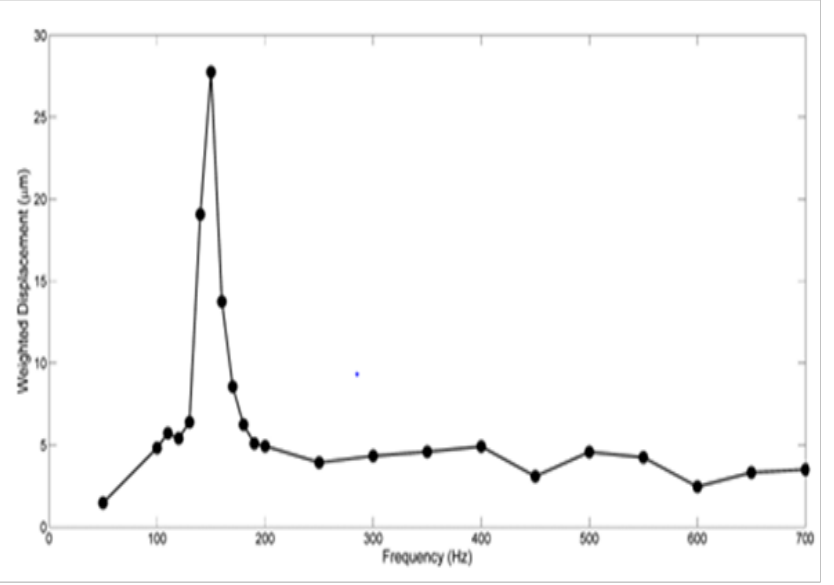

Figure $\mathbf{5}$ Vibrational analysis of decellularized dermis. Determination of the resonant frequency from measurement of the maximum displacement of a sample vibrated between 0 and $100 \mathrm{HZ}$. Note the maximum displacement is measured and converted into a modulus obtained from vibrational studies using the calibration curve shown in Figure 6.

\section{Mechanotransduction in collagenous tissues}

Collagen fibrils are a major factor in the conversion of mechanical forces and work into stored energy; in many cases, this energy is stored in the form of high molecular weight polymers such as collagen fibers. ${ }^{2}$ When muscles and tendons are loaded, the muscle does work on the collagen fibers in tendon and energy is stored as work in the tendon. During tendon stretching, the applied force results in work stored elastically, by stretching the flexible regions of the triple helix. This energy is released after the load is removed and transmitted to the muscle or the energy is stored in the form of high molecular weight proteins via a process termed mechanotransduction.

Collagen fibrils are attached to cell membranes through attachment molecules such as integrins and other cell surface macromolecules.
During stretching of the collagen fibers, tension is transmitted through the cell membrane setting into motion the activation of the phosphorelay pathways inside the cell. ${ }^{2}$ Tension typically activates the MAP kinase pathways leading to synthesis of new collagen to "bolster" the ability of the tendons and other tissues to support loads. ${ }^{2}$ Thus, some of the mechanical energy generated from the muscle tension is stored in the form of high molecular weight polymers; energy is stored in the form of covalent bonds that link amino acids together in the newly synthesized collagen fibers. When external tensile loads decrease, the collagen fibers are resorbed and energy is released from the broken covalent bonds. This can occur during nonloading events, such as prolonged bed rest or when an astronaut is in a reduced gravitational field for a prolonged time period.

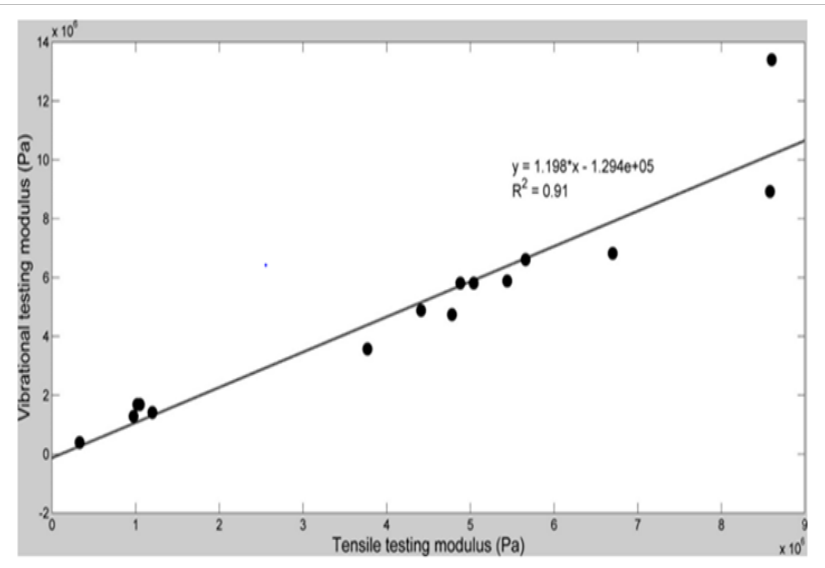

Figure 6 Calibration curve of the modulus determined from vibrational studies (obtained from measuring the frequency at which the maximum displacement occurs) versus the tensile modulus determined from force versus extension curves for decellularized dermis and silicone rubber. The tensile modulus was measured from the slope of incremental stress-strain curves and the modulus from vibrational studies was obtained from the resonant frequency as described by Shah et al.

In this manner, collagen fibers are dynamic structures that are constantly growing or resorbing depending upon the level of tension they experience. The interactions between the collagen fibers and cells in tissues are an exciting part of the dynamics that occur in ECM biology and ultimately affect health and the pathogenesis of disease processes such as cancer.

Recent advances in the understanding of integrin mediated mechanotransduction ${ }^{20,21}$ are important to understand the relationship between external mechanical loading and the events that occur at the interface between the ECM, surrounding cells and any implant that is in contact with the cell-ECM interface. This suggests that any interruption of integrin mediated binding to the surrounding ECM at the implant-tissue interface has consequences in terms of modifying mechanotransduction and the cellular response to allografts and implants.

Integrin mediated mechanotransduction plays an important role in development and tissue homeostasis. ${ }^{20}$ After implantation of an allograft or tissue engineered scaffold, cell binding occurs. ECM receptors such as integrins facilitate assembly of different ECM components into a variety of geometrical form. ${ }^{22}$ Specifically integrin $\alpha$ and $\beta$ subunits bind to type I collagen receptors ${ }^{5}$ and lead to a conformational change in the integrin subunits (Figure 7). Integrins then recruit numerous proteins to their cytoplasmic tails forming 
nasant adhesions some of which stabilize and form focal adhesions. Focal adhesion maturation requires further integrin clustering, intracellular F-actin binding and reinforcement of linkages between actin and actomyosin. Activated integrins are coupled to F-actin through actin binding proteins including talin and vinculin, These molecules are involved in the "molecular clutch" that is responsible for cell movement as a result of actin flow inside the cell as F-actin is polymerized leading to force generation and propulsion of the cell body. ${ }^{20}$

Phosphorelay pathways can be activated not only by integrin binding to ECMs, but by direct stretching of the following: cell membranes, ion channels, intracellular junctions, and growth factor and hormone receptors. Activation of these moieties leads to changes in protein synthesis, altered gene expression and cell mitosis. ${ }^{19}$ (Figure 7). All these events occur at the cell-allograft and cell-tissue engineered material interface and dictate rate, composition and extent of tissue in growth. Providing the correct boundary forces and stresses to equilibrate the implant-tissue interface is important to prevent cellular hyperplasia and excessive collagen deposition.

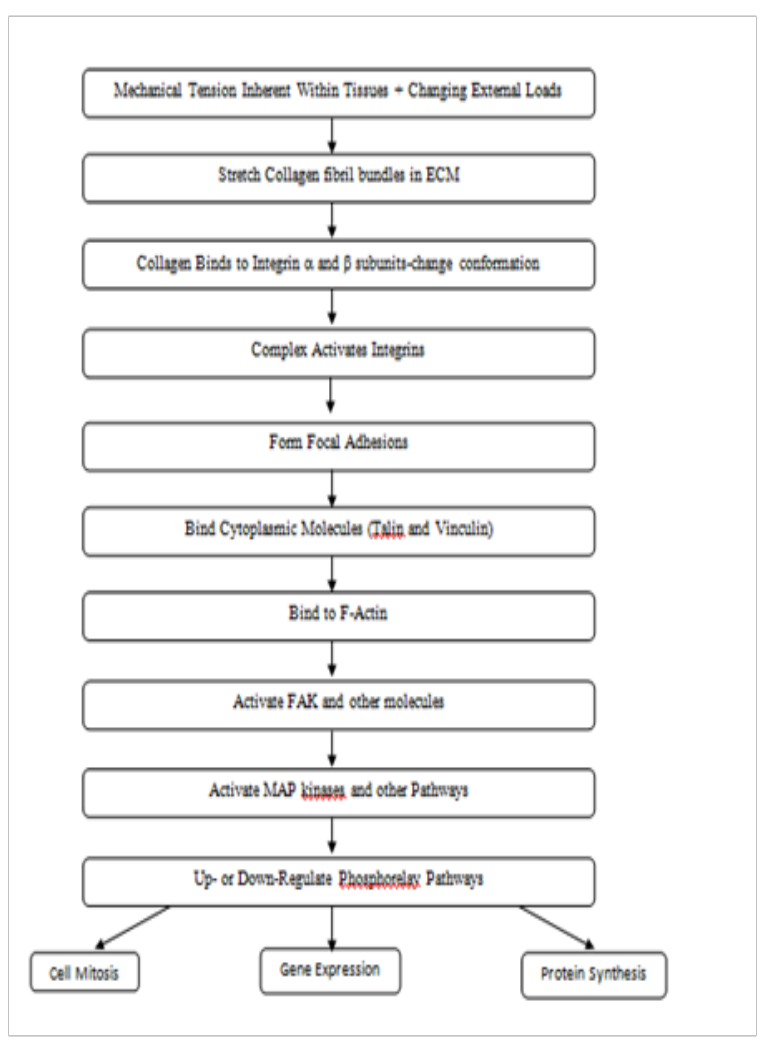

Figure 7 Diagram illustrating how mechanical tension exerted in tissues, such as the tension in Langer's lines in the skin, affects cellular behavior through activation of the phosphorelay pathways. Integrin and non-integrin dependent pathways are involved in activating or deactivating protein synthesis and gene expression because of changes in the mechanical loading experienced by the ECM. In integrin dependent mechanotransduction, tension in collagen fibers causes a conformational change in the integrin subunits that are attached to specific bands on the collagen fibrils. This causes formation of focal adhesions, binding of talin, other intracellular molecules including F-actin. This leads to activation of FAK in the cytoplasm followed by activation of MAP kinase pathways leading to alteration of protein synthesis and gene expression. For example, increased muscular work by weight lifting, or mechanical forces on the palm of the hand associated with pounding a hammer, cause skin cells to up-regulate protein synthesis and cell mitosis. This causes more skin covering the muscles and thicker skin on the palm of the hands, respectively.

\section{Conclusion}

Collagen fibers are the structural elements found in vertebrate tissues that transmit forces, store and dissipate energy. Collagen fibers limit the deformation of tendon and other load bearing tissues and have a hierarchical structure that includes collagen molecules, microfibrils, fibrils, fibers and fascicles. Collagen molecules are packed into a quarter-stagger packing pattern with neighboring molecules staggered by multiples of $\mathrm{D}$, which is about $22 \%$ of the molecular length. During mechanical deformation, collagen molecules are stretched as well as the gap region of the $\mathrm{D}$ period. The elastic modulus of collagen molecules can be calculated from the slope of the tensile stress-strain curve after correction for, viscous slippage, the collagen content and the change in axial rise per amino acid residue that occurs during tensile deformation. In tissues such as tendon, the low modulus region occurs due to unfolding of the crimped collagen fibers. The differences in the values of the modulus measured reflect differences in collagen concentration, orientation and the presence of other materials. The low modulus region involves geometric alignment of collagen fibers with the tensile axis as well as stretching of other components in series with the collagen fibers. At larger strains, collagen molecules and fibrils slide by each other, which lead to energy losses and viscous effects.

Mechanical loads applied externally to collagen fibers in ECMs, integrin sub units and other binding elements play important roles in mechanotransduction and events that occur at the implant-host interface (Figure 7). Excessive stress at the interface can lead to intimal hyperplasia and excessive ECM deposition ultimately leading to implant failure or encapsulation. It is hoped that measurement of the mechanical properties of tissues and implants in vivo will help to better understand collagen fiber behavior in health and disease and to prevent stress concentrations at the interface between host tissues, allografts and tissue-engineered materials.

\section{Acknowledgements}

None.

\section{Conflict of interest}

The author declares no conflict of interest.

\section{References}

1. Silver FH, Landis WJ. Viscoelasticity, energy storage and transmission and dissipation by extracellular matrices in vertebrates. In: Fratztl P editor, Collagen. Structure and Mechanics. New York, USA: Springer; 2008. p. 133-154.

2. Silver FH. Mechanosensing and mechanochemical transduction in extracellular matrix. biological, chemical, engineering and physiological aspects. New York, USA: Springer; 2006. 292 p.

3. Silver FH, Quintero L. Comparison of the histologic responses observed at the arterial puncture site after employing manual compression and a new collagen to achieve hemostasis. Cath Lab Digest. 2003;11(9):52-58.

4. Silver FH, Kato YP, Ohno M, et al. Analysis of mammalian connective tissue: Relationship between hierarchical structures and mechanical properties. J Long Term Eff Med Implants. 1992;2(2-3):165-198.

5. Silver FH, Freeman JW, Seehra GP. Collagen self-assembly and development of matrix mechanical properties. $J$ Biomechanics. 2003;36(10):1529-1553.

6. Silver FH, Shah R. Measurement of mechanical properties of natural and engineered implants. Advances Tissue Eng Regen Med Open Access. 2016;1:1-9. 
7. Ricard Blum S. The collagen family. Cold Spring Harb Perspect Biol. 2011;3(1):a004978.

8. Hulmes DJS. Collagen diversity, synthesis and assembly. In: Fratzl P editor, Collagen. Structure and Mechanics. New York, USA: Springer; 2008. p. $15-47$

9. Yamada H. Strength of biological materials. Baltimore, USA: Williams, Wilkins; 1970. 159 p.

10. Fung YC. Biomechanics: mechanical properties of living tissue. 2nd ed. NY, USA: Springer; 1973. 568 p.

11. Dunn MG, Silver FH. Viscoelastic behavior of human connective tissue: relative contribution of viscous and elastic components. Connect Tissue Res. 1983;12(1):59-70.

12. Shah R, Pierce MC, Silver FH. A method for non-destructive mechanical testing of tissues and implants. J Biomed Mat Res Part A 2017;105(1):5-22.

13. Torp S, Baer E, Friedman B. Effects of Age and of mechanical deformation on the ultrastructure of tendon, Proceedings of the Colston Conference. $1974 ; 26: 223-250$

14. McBride DJ. Hind limb extensor tendon development in the chick: $A$ light and transmission electron Microscopic study. M.S. Thesis in Physiology. Rutgers University, USA: Spinger; 1984.

15. McBride DJ, Hahn R, Silver FH. Morphological characterization of tendon development during chick embryogenesis: Measurement of birefringence retardation. Int J Biol Macromol. 1985;7(2):71-76.
16. McBride DJ, Trelstad RL, Silver FH. Structural and mechanical assessment of developing chick tendon. Int J Biol Macromol. 1988;10(4):194 200 .

17. Sasaki N, Shukunami N, Matsushima N, et al. Time-resolved X-ray diffraction from tendon collagen during creep using synchrotron radiation. J Biomech. 1999;32(3):285-292.

18. Folkhard W, Geercken W, Knorzer E, et al. Structural dynamic of native tendon collagen. J Mol Biol. 1987;193(2):405-407.

19. Silver FH, Siperko LM. Mechanosensing and mechanochemical transduction: How is mechanical energy sensed and converted into chemical energy in an extracellular matrix. Crit Rev Biomed Eng. 2003;31(4):255331.

20. Sun Z, Guo SS, Fassler R. Integrin-mediated mechanotransduction. J Cell Biol. 2016;215(4):445-456.

21. Case LB, Waterman CM. Integration of actin dynamics and cell adhesion by a three-dimensional mechanosensitive molecular clutch. Nature Cell Biol. 2015;17(8):955-963.

22. Gasiorowski JZ, Murphy CJ, Nealey PF. Biophysical cues and cell behavior: the big impact of little things. Ann Rev Biomed Eng. 2013;15:155176. 